\title{
Computer Manipulatives in an Ordinary Differential Equations Course: Development, Implementation, and Assessment
}

\author{
Haynes R. Miller, ${ }^{1,3}$ and Deborah S. Upton ${ }^{2}$
}

The d'Arbeloff Interactive Mathematics Project or d'AIMP is an initiative that seeks to enhance and ultimately transform the teaching and learning of introductory mathematics at the Massachusetts Institute of Technology. A result of this project is a suite of "mathlets," a carefully developed set of dynamic computer applets for use in the university's ordinary differential equations course. In this paper, we present the rationale for such computer innovations, the philosophy behind their design, as well as a discussion of their careful development and implementation. Survey results are reported which yielded positive student feedback and suggestions for improvement.

KEY WORDS: transfer; undergraduate mathematics education; differential equations; simulation; applet

\section{INTRODUCTION}

At the opening of the current millennium, the Massachusetts Institute of Technology experienced a very exciting cycle of educational renewal and experimentation. The d'Arbeloff Interactive Mathematics Project, d'AIMP, created a score of computer "manipulatives" or "mathlets," small, self-contained, single-purpose dynamic learning environments, for use specifically in the principal undergraduate differential equations course. These learning objects are now rendered in Java and are freely available on the Web at $<$ http://www-math.mit.edu/daimp $>$.

In this paper we present the rationale for such computer-based innovations, the philosophy behind their design, and a discussion of their development, implementation, and assessment.

\footnotetext{
${ }^{1}$ Department of Mathematics, Massachusetts Institute of Technology, Cambridge, MA, 02139, USA

${ }^{2}$ Department of Mathematics, Stonehill College, Easton, MA, 02357, USA

${ }^{3}$ To whom correspondence should be addressed;

E-mail: hrm@math.mit.edu
}

The section "Institutional Background: Three Dilemmas" describes the historical background for this work, reviewing the specific conditions at MIT in which it was conceived and carried out. These institutional conditions pose a series of challenges to the teaching of basic mathematics, which are formulated as three dilemmas.

The section "Intellectual Background: The Case for Mathlets" discusses the intellectual background for this work: modes of feedback, modes of transfer, modes of instructional support, and a review of similar work. This section seeks to provide a review of the current state of the literature and courseware development rather than paint a picture of what was known, or known to us, when the project began. The section ends with a summary of the design considerations informing the creation of these mathlets, and a description of previous work that ours builds on.

The section "Design Considerations and Three Examples" provides examples of three of the mathlets, chosen to show how they address our three dilemmas. The section "Design Cycle and Assessment" discusses the design cycle and describes our assessment methods and results. A "Conclusion" briefly summarizes our findings, and assembles some 
general principles we have learned about the use of technology in education.

\section{INSTITUTIONAL BACKGROUND: THREE DILEMMAS}

Since its inception in 1861, MIT has espoused the principle that technical education should be based on broad scientific and mathematical principles. The connected and dynamic character of the MIT undergraduate program results in part from this overall policy decision. Students are accepted by MIT as a whole rather than by an individual school or department, and the faculty as a whole share responsibility for their education. A specified list of science and mathematics courses (consisting of two semesters of calculus, two semesters of physics, and one semester each of chemistry and biology) is required of all undergraduates. In addition, students are required to take two restricted electives in science and technology (RESTs). Approximately $80 \%$ of each class elects to take Differential Equations, 18.03, as a REST. This course is required as part of the major by most departmental programs, and there has been an increase in the number of life science and social science majors taking the course even though it is not required by those majors.

This policy choice, which has been emulated by many technologically oriented universities across the country, confronts the providers of basic science and mathematics courses with a complex challenge. They are charged with conveying fundamental principles of science and mathematics to freshmen (and sophomores, to a lesser extent) who come from widely diverse backgrounds and have widely diverse educational goals. These students are eager take courses in the field they came to study, or to experiment with courses in subjects not represented in high school. Many students have a central interest in mathematics or physics per se, but two thirds of MIT undergraduates pursue an engineering degree. Typically they take their first engineering course in their third semester. At the same time, the science and mathematics "service courses" tend to be less well funded by the institution than do disciplinary courses, and host departments respond by teaching them in large lectures accompanied by recitations. ${ }^{1}$

\footnotetext{
${ }^{1}$ The MIT Physics Department has moved to a Studio Physics delivery system for much of their core science teaching. This approach still puts around 100 students in the classroom.
}

The core science courses, furthermore, have a dual obligation. They are expected to provide students with an understanding of the modes of analysis characteristic to the subject of study, as part of the students' preparation as engaged and technologically informed citizens, while also preparing students with skills and knowledge which will be called upon in later disciplinary work. In a school like MIT, one which is "polarized around science" (Killian, 1949), this second task is of particular concern in mathematics.

We formulate the challenges resulting from these institutional conditions as three dilemmas.

Facilitation of Transfer. This institutional background presents a challenge to those teaching basic mathematics, and in particular differential equations: how can the operations and content of differential equations be brought to life in a way that has clear connections with downstream courses while preserving the desirable generality of the mathematics? The importance of this task is reflected in the perceptions by engineering faculty that their students are not well prepared (Miller and Upton, 2002; Willcox and Bounova, 2004). It is further confirmed by students who claim that they have forgotten underlying principles that were taught in their freshman year or that they never actually understood the ideas when first presented (Nasr et al., 2003; Upton, 2001). However, despite much anecdote, there are surprisingly few quantitative studies of transfer of mathematical skills and understanding into engineering contexts in the literature.

Examples versus Theory. In teaching mathematics, especially to students who do not intend to pursue mathematics professionally, one is continually faced with another dilemma. Simply stated, one must either present a general case with symbols or present an example with numbers. Both extremes are deficient. The general theory is at the start meaningless to the student, while on the other hand any example generalizes in diverse ways and so is confusing to the student. Good teachers rely on their experience to visualize the range of specializations and thus bring the theory down to earth. At the same time, teachers need to give significance to the examples by understanding what in the example is essential and what is not. These understandings are difficult and timeconsuming to convey using static media such as chalk, slides, or pages of a textbook.

Algorithm versus Concept. Another well-known weakness of standard mathematics courseware, especially in engineering oriented differential equations 
courses, is that it tends to be based on algebraic manipulation of symbols. To the teacher, these symbols carry significance, even graphical significance, but students have no way of making that connection. Typically symbolic expressions lend themselves to procedural operations, while graphical representations link more easily with conceptual understanding.

\section{INTELLECTUAL BACKGROUND: THE CASE FOR MATHLETS}

This section reviews the current state of the educational theories that underlay the creation of the d'AIMP mathlets and summarizes the design considerations informing the creation of the mathlets. We end with a listing of the specific design considerations we were led to.

Modes of feedback. In Rethinking University Teaching (2002), Laurillard considers a variety of distinct media used in teaching. Among other forms she discusses adaptive media, which she defines as "the computer-based media capable of changing their state in response to the user's actions" (p. 126). Under this heading, she defines a simulation as "a program that embodies some model of an aspect of the world, allows the user to make inputs to the model, runs the model, and displays the results." A further classification, due to Allessi and Trollip (2001), locates the mathlets as iterative simulations, which (as described by Lipson (2006), p. 2) "allow learners to select different parameters to permit observation of the phenomena [being studied] under different conditions."

Laurillard stresses that "action without feedback is completely unproductive for a learner" (p. 55). "Feedback is critical to the learning process, as every theory of learning acknowledges, from behaviourist to social constructivist.... Feedback on students' action is the weakest link in the traditional educational process" (p. 126). She is consequently interested in the potential of media to effect feedback to the student.

She distinguishes between two types of feedback: intrinsic and extrinsic. Intrinsic feedback is a response to an action that is inherent in the system in which the action took place. Extrinsic feedback is a response to an action from outside the system. Praise, encouragement, and warnings, may all be useful forms of feedback in an educational context, but they are extrinsic to the subject the learner is grappling with, and, to that extent, they distract the attention of the learner from his or her primary task. Intrinsic feed- back informs the learner of a property of the system under consideration, and represents the most direct way this information can be conveyed. As Laurillard says, "The informational content of intrinsic feedback is extremely valuable to the learner.... It is individualized, private, formative feedback, which helps to build their understanding of the internal relations between theory and practice" (p. 127).

Laurillard contends that "The ability to offer intrinsic feedback is unique to the computer, and forms the core of any understanding of the contribution that ICT [Information and Communications Technology] can make in education..." (p. 126).

Modes of transfer. Since facilitation of transfer is one of the primary objectives of this work, it is worthwhile considering what is meant by "transfer." Bransford and Schwartz (1999) provide a useful critique of research on transfer that tends to "produce assessments that make people "look dumb"" (Schwartz et al., 2005). They distinguish between two types of transfer assessment. The first tests proficiency at what they call SPS, Sequestered Problem Solving; this is the common approach, employed for example by Adamczyk et al. (2002). A deeper understanding of the meaning of transfer leads to assessments of how well it succeeds as Preparation for Future Learning, or PFL. Such assessments will measure the ease of learning in new contexts with or without the experience from which transfer is being studied.

Schwartz et al. (2005) continue this analysis by pointing out that when viewed as PFL, transfer has at least a two-dimensional space of attributes: "efficiency" and "innovation." Assessments of transfer success typically measure only the efficiency coordinate and make no attempt to evaluate the improvement of the ability of the learner to come up with appropriate questions or plans of action. These authors comment that in fact "Experimental studies show that [high] efficiency can often produce 'functionally fixed' behaviors" (p. 29) and so is of questionable value. They envision the learning process as moving along a trajectory within this space, and identify an "optimal adaptability corridor" in which the two aspects of transfer are in approximate balance. They recognize that some learners' trajectories form curved arcs on one side or the other of the diagonal.

These considerations contribute to our view that transfer, properly interpreted, is enhanced by providing students with conceptual hooks as well as experience with re-usable algorithms. The advantages of an appropriate level of abstraction in establishing 
flexible transfer are well articulated in the classic National Research Council study edited by Bransford et al. (1999).

Modes of instructional support. Lipson (2006) provides a convenient typology of instructional environments, with reference to their use in supporting learning with simulations. " $\ldots$ instructional supports can be classified into three types: (1) directed - they tell the learner what to do by providing assignments; (2) guided - they provide a little direction by pointing out some relevant aspects to examine; and (3) inquiry-based - they ask questions, rather than providing any direction." (p. 6). Assignments provide an example of a directed support; "Assignments are small exercises that can help students identify the important variables in the simulation, develop hypotheses, and interpret the results" (p. 6). Model progressions exemplify guided supports; these "structure the simulation so students do not deal with the simulation in its full complexity initially. Learners are initially given a simplified version of the model and are introduced to more complex versions in a series of stages." (p. 6). Inquiry-based support leads to a form of 'discovery learning.'

Lipson reports on a substantial body of research indicating that in general inquiry-based support of simulations does not work well unless it is provided in a group setting. Mayer (2004) makes the same point more strongly. A balanced approach, with carefully specified sequenced objectives - a model progression broken down into connected assignments-often showed best results.

Review of similar work. The d'AIMP mathlets were based on an earlier suite of programs, Interactive Differential Equations < http://www.aw-bc.com/de $>$, authored by Beverly West, Steven Strogatz, Jean Marie McGill and John Cantwell, and designed by Hubert Hohn. These programs were discussed by McDill et al., (1997). An accompanying paper workbook provided instructional support (West et al., 1997). The d'AIMP project allowed us to bring this prior work up to a contemporary standard of performance, bring it into better accord with our curricular choices at MIT, add many new tools to the kit, and increase interactivity.

Another project close in spirit to the d'AIMP work is MathinSite (http://mathinsite.bmth.ac.uk/index.html). Developed mainly by Peter Edwards at Bournemouth University, UK, MathinSite consists in a collection of two dozen narrowly focused simulations (like ours, rendered first in Basic and later ported to Java). Some are accompanied by a "Theory Sheet" describing the underlying mathematics, or by a "Work Sheet," containing a model progression leading through the functionality of the applet to illustrate the mathematical points it captures. Typically there are cursor-controlled parameters, numerical readouts, and color coding. There is often a menu of functionality options, so each tool tends to be more multipurpose than ours are. The skin is closer to Java standard than ours. One interesting and striking design decision was to make the principal graphing window a moveable sheet of quadrille paper. This allows various parameters to achieve a wider range of values than we can accommodate, and gives the tools a pleasingly open look with an engineering feel to them. This work has been frequently reported on; see for example Edwards and Edwards (2003).

Beyond these, there is an explosion of web-based mathematical visualization aids of all sorts. Many are collected in peer-reviewed archives, such as the Mathematical Association of America's Mathematical Sciences Digital Library, MathDL < http:// mathdl.maa.org/>, which houses the JOMA Mathlet collection and provides standards and protocols for the production of applets in their Mathlet Developers' Area. MERLOT, the Multimedia Educational Resource for Learning and Online Teaching $<$ http:// www.merlot.org/Home.po $>$, provides a similar environment for higher education in general. The National Science Digital Library's Educational Resources for Science \& Mathematics, iLumina $<$ http://www.ilumina-dlib.org/ $>$ is another archive focusing on science. While the work found in these archives is often of excellent quality, none of it shares the goals of our work as closely as MathinSite, and we will not review it in more detail.

\section{DESIGN CONSIDERATIONS AND THREE EXAMPLES}

The mathlets were built with the following design features in mind:

(1) Each mathlet addresses a precisely targeted concept, minimizing the complexity of the tool and maximizing the specificity of the illustration. A selection of parameter values is carefully chosen to maximize the clarity of important features. When bundled together, the suite of applets offer the advantages of a more general tool without sacrificing precision and ease of use.

(2) In each mathlet, information is represented in a variety of ways - pictorially, graphically, 
numerically, and symbolically. The different views and their connections allow for more questions and thus offer a more robust understanding of the target concept. Positioning and color-coding reinforce the relations between these various representations.

(3) Each mathlet is a manipulative rather than an animation. Each incorporates a high degree of interactivity that is meaningful for learning and can aid in retention. Many web pages offer buttons to click but have no engaging, challenging content with which to interact. With these tools, the user must select parameter values, by cursor movement on a window, setting sliders, or clicking on radio buttons, and can make measurements of objects in graphing windows. The representations are linked in real time so that a change in one produces corresponding real-time changes in all the others.

(4) Information is often displayed in a progressive manner, controlled by toggles.

(5) The technology is easy to use. Unlike computer algebra packages and graphing calculators, students learn to use them very quickly, minimizing time deflected away from the essential mathematics content. Further, the various mathlets share a common set of conventions.

(6) The mathematics underlying each mathlet is scrupulously correct. This is essential because the applets are designed not merely to give a vague visual correlate to the "important" algebraic manipulations, but, also, and more powerfully, to provide experimental data which the student may measure and compare with the theory.

(7) "Help" pages specify the various functionalities of each tool.

Each of the following examples of the d'AIMP mathlets illustrates our solution to each of the three dilemmas outlined above.

Facilitation of Transfer: Amplitude and Phase. The mathlet "Amplitude and Phase: Second Order", deals with a standard simple mechanism - a mass driven by a piston attached to a spring and damped by a dashpot. The motion of the mass is described by a second order linear-time invariant (LTI) equation. This tool considers a standard sinusoidal forcing motion. It allows the student to vary the spring

\footnotetext{
${ }^{2}$ This mathlet and the Linear Phase Portrait mathlet build on earlier IDE tools (Interactive Differential Equations, 1997).
}

constant, the damping constant, and the input frequency. The mechanical system itself is displayed, along with a graph of the motion of the piston and of the mass. Lines on the graphing window indicate the time lag, and readouts give the values of the period and time lag. Position variables are displayed when the cursor is rolled over the graphing window. A button animates the whole system, and the motion along the graphed solution is indicated. A toggle activates a second set of windows, showing the amplitude and phase response and the complex frequency response. As the various system parameters are adjusted, the whole configuration adapts to the current values (See Figure 1).

Figure 1 represents a screen capture of a state of this mathlet. When it is first opened, the three windows at the right and the two equations below them are absent: much less information is displayed. In getting to the displayed state, the user has set values of the spring constant $k$, the damping constant $b$, and the circular frequency $\omega$ of the plunger motion; clicked the $[>>$ ] key to animate the spring; and having understood how the position of the plunger and of the mass are represented by graphs in the central window - assisted by the color coding - varied the circular frequency through a range of values, and observed the resulting variation in these graphs. Finally, the user has clicked the [Bode and Nyquist Plots] key to reveal the right hand column. Now the user can see graphically how the salient characteristics of the solution - its amplitude and its phase lag-vary with $\omega$, and that these two parameters are captured as the magnitude and argument of a certain complex number which varies with $\omega$ as show in the bottom left window.

These graphical elements carry complete information about the periodic system response, and form the core of a large fragment of later engineering coursework. They also represent faithfully the manner of solution taught in the mathematics course. This tool is re-used in down-stream engineering courses, building a visual and conceptual bridge to the prior mathematics course. It allows the upstream mathematics course to touch on parts of the subject - frequency response - which are often regarded as too sophisticated for this level of student because they seem mysterious when expressed in formulas. The tool reinforces the lesson that sinusoidal motion is determined by a very few parameters - frequency, amplitude, and phase. This allows the student to view the periodic solution as an object, which can be controlled by the parameters of the system. This 


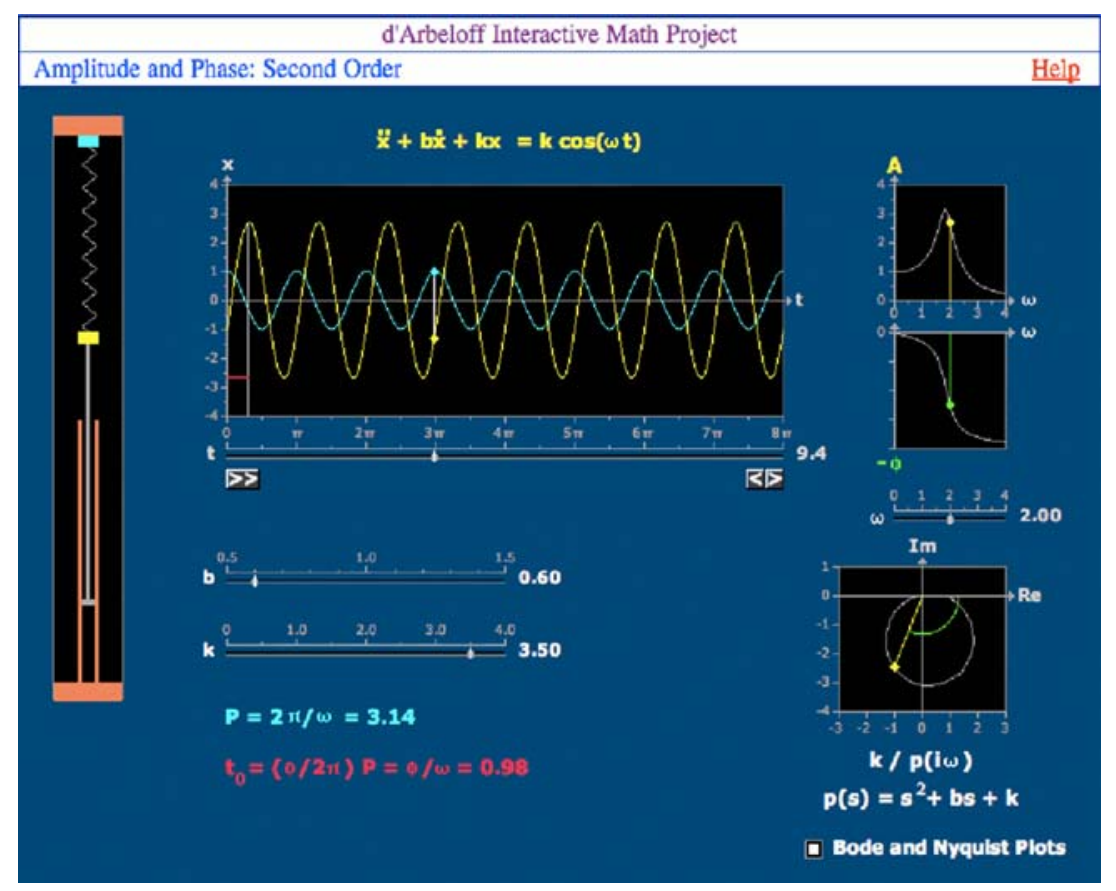

Fig. 1. Amplitude and phase, second order.

conceptual step, from viewing a function as a rule (understood by means of evaluations) to an object (understood by means of determining parameters) is a fundamental learning objective in a differential equations course.

Example versus Theory: Linear Phase Portraits. The d'AIMP mathlets "Linear Phase Portraits: Cursor Entry" and "Linear Phase Portraits: Matrix Entry" illustrate a solution to the dilemma of choosing between presenting general and special cases. These applets allow investigation of the variation of solution trajectories as the parameters are changed in a homogeneous system of first order linear differential equations. The textbook story is that in most cases the trace and determinant determine the essential characteristics of the phase portrait. In traditional accounts, one does a few examples each of which seems isolated, and students have no way of knowing how the image morphs from one example to another. With this tool one can control the trace and determinant using either sliders or cursor movement over the trace-determinant plane. The critical parabola separating distinct behaviors is unmistakable. A large graphing window displays a few trajectories. Different areas of the trace-determinant plane are colored differently, and representative trajectories are drawn in the same color. Additional trajectories can be produced by clicking on the graphing plane. Within a given trace-determinant class there are still two degrees of freedom. In the cursor entry Mathlet, this variation can be controlled by cursor movement or slider adjustment in a different window. This allows excellent visualization of the diversity of shapes within a given class. In the matrix entry tool, a toggle allows a choice between selecting the companion matrix with the given trace and determinant-in which case the matrix is chosen by setting the trace and determinant - or, alternatively, controlling the matrix by directly altering the matrix entries. Another toggle opens or closes a window displaying the eigenvalues of the matrix along with a read-out of their values (See Figure 2).

In homework use of the Matrix Entry tool, we typically ask the student to set fix three of the four entries in the matrix at specified values, and then vary the fourth. The trace-determinant pair moves along a straight line passing through several regions, corresponding to different phase portrait types. The student observes the deformation of the trajectories and can understand how a spiral degenerates to a defective node and re-emerges as a proper node. The critical values of the varying matrix entry are then 


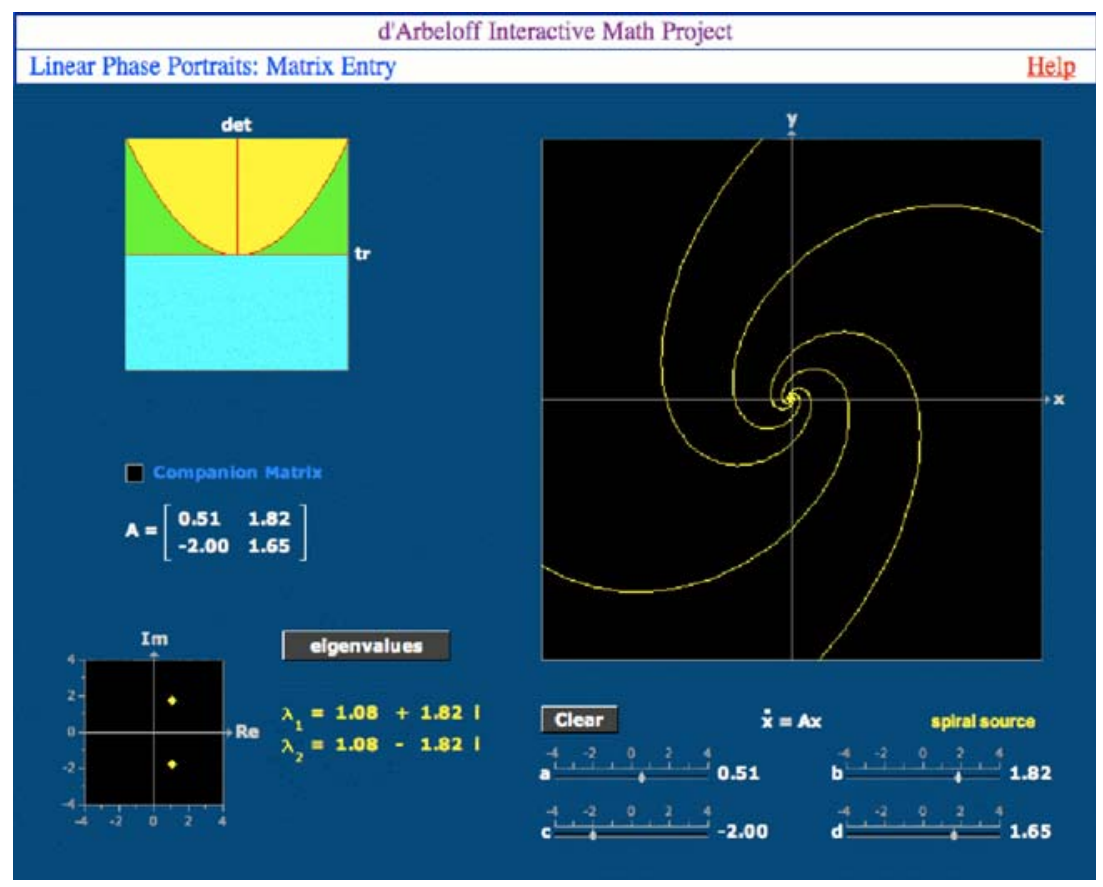

Fig. 2. Linear phase portraits, Matrix entry.

requested; the student is asked to name the various phase portrait types and sketch representative examples of them.

Algorithm versus Concept: Fourier Coefficients. Our solution here is to require students to carry out experiments using the graphics, measuring parameters and correlating their measurements with the theory. Although this works very well with the Amplitude and Phase mathlet, we choose to discuss "Fourier Coefficients." In this applet, a column of sliders allows the user to set the values of Fourier coefficients. The resulting finite Fourier sum is graphed. When the user moves a slider, a representation of the corresponding sinusoid is also shown, developing an intuition, which is usually missing in students at this level, about adding functions. Further buttons display a target function, which the user attempts to approximate by choice of Fourier coefficients. Radio buttons show or hide formulas for the Fourier sum and the root mean square distance between the target and its Fourier approximation (See Figure 3).

This mathlet aims at instilling a conceptual understanding of Fourier coefficients-the coefficients that result in optimal approximations as opposed to the standard procedural definition of certain integrals. The student can discover the values of the coefficients by this graphical approach and then verify this discovery analytically using a computational algorithm. The mathlet also illustrates concepts, such as orthogonality, which go somewhat beyond what one can teach at this level, and thereby offers the opportunity to enhance transfer by reference to a familiar visual context.

\section{DESIGN CYCLE AND ASSESSMENT}

The early development phase. Work on creating these manipulatives began in Fall 2000. They were initially written in True Basic. In February 2002 they were used in homework and for classroom demonstrations in a large differential equations class. They were presented as executables and the students had to use them in on-campus computer clusters. In Fall 2002 the second author joined the team and began an intensive formative assessment of the manipulatives as they existed at the time. This resulted in numerous improvements that were incorporated in time for use in homework assignments in Spring 2003. An extensive survey and interview study was conducted with students to learn about the way in which they used the manipulatives in that class. These data led to a better understanding of how students responded to this pedagogical medium. The manipulatives have 


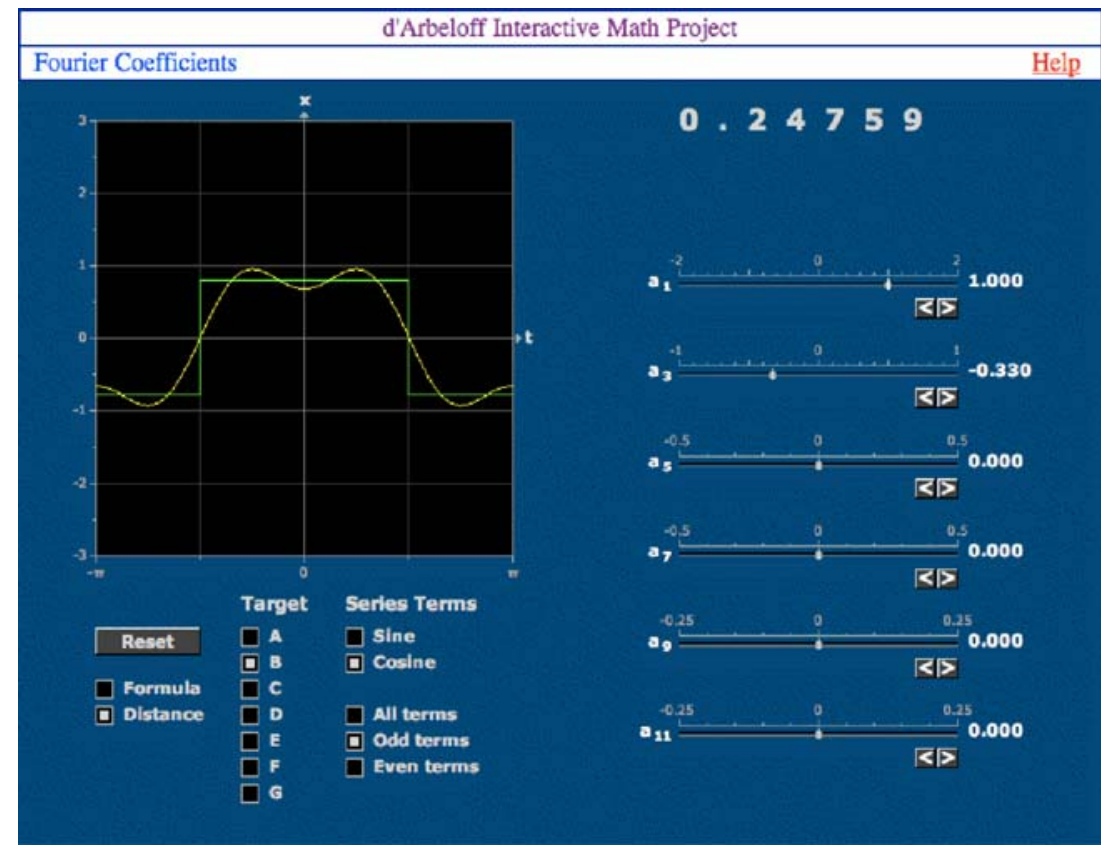

Fig. 3. Fourier coefficients.

been a staple in this course since then. In Spring 2002 a programmer began porting the code to Java, with a second programmer completing the project by Fall 2005.

During the development and implementation phases of the project, a close collaboration was achieved between teacher, software designer, programmer, and assessment expert. This added greatly to the efficiency of the product and enjoyment of the process. Involving the students at an early stage in usability testing was critical as well.

Initial Usability Testing. The initial stages of the evaluation began with usability testing of the mathlets. The sample for this testing consisted of eight students from an introductory ordinary differential equations course, four men and four women, with differing levels of mathematical ability. None of the participants had any prior exposure to the Mathlets. Each participant was required to meet with the researcher for about an hour every other week during the semester. At each meeting, the participant was provided with a computer that gave him or her access to a specified applet, and a script to guide the student through its use. Each script consisted of a sequence of questions asking the student to describe what he or she observed, how the screen images changed in response to specified actions such as moving a slider, and what the various images on the screen repre- sented mathematically. It also contained fragments of assignment sequences, testing reactions to various possible homework usages. The researcher read the script aloud and each participant was asked to think aloud as he or she followed the given instructions. The computer screen was videotaped and the verbal exchange was recorded as the participants worked, and this record was later analyzed. Based on observation of these results, improvements were made to the manipulatives and their scripts and the testing cycle was repeated.

The usability testing amounted to a formative assessment of the applets before their full implementation. Bugs in the programs were discovered and rectified, and obscurities were clarified. Some of the mathlets were found to present too much information on the screen at one time for students to absorb comfortably. In response, these mathlets were revised so parts of the display were hidden at first, requiring the user to invoke them by pressing a toggle. The student's thinking process was followed as it led him or her to use the applet to achieve a richer understanding of the mathematics. Students who habitually relied on only algebraic understanding became less reluctant to use geometric means by using the manipulatives. We also learned how to ask the students to interpret various graphical elements in the mathlets, as a way of leading 
them to think about the graphical meaning of the mathematics.

Later Usability Testing and Assessment. With improvements made and a better understanding of the behavior and thinking processes of the students achieved, the applets were introduced into an introductory Differential Equations course. Homework assignments, which can be found on the project's website, (http://www-math.mit.edu/daimp), included investigations with the following sequence of mathlets-Phase Lines, Complex Roots, Amplitude and Phase: First Order, Damped Vibrations, Amplitude and Phase: Second Order, Beats, Fourier Coefficients, Convolution: Accumulation, Convolution: Flip and Drag, and Linear Phase Portraits: Cursor Entry. (During this time, many of the applets were identified by other names. The names of the currently available variants of these prototypes are being used for convenience.) A representative sample of 60 students was administered web-based surveys to complete after the use of each manipulative. The surveys provided both quantitative and qualitative information about how students learned to use visuals, how visuals contributed to their understanding of the material, how they contributed to students' enjoyment, and ways in which these tools can be successfully used in problem sets.

Survey findings. Each survey asked the participants to rate the overall ease of learning to use the mathlet. For the first five tools, the participants were asked to give a whole number rating on a scale from 1 to 5 , with 1 being "very hard" and 5 being "very easy." For the next five tools, a 1 to 7 scale was used, with 1 being "very hard" and 7 being "very easy." This change was made in an effort to allow for more variability as well as correspond to the rating system students were already familiar with from university course evaluations. Fifty-nine, 55, 55, 56, 56, 54, 52, 47, 47 and 46 responses were received for the tools listed above respectively. Table 1 shows the breakdown of these responses in terms of the quantity of each whole number rating received for each tool.

The data shown indicates that for each mathlet, more than 50 percent of the participants gave the first five tools a rating of 4 or 5 and the last five tools a rating of 5,6 or 7 . Thus, more that 50 percent of the participants found the overall ease of learning to use the visuals "easier than average."

We were interested in how the manipulatives interacted with students' personal educational support structure, and asked the participants to respond to the question: "If you needed help [in using the mathlet], where did it come from?" The number that reported needing help consistently decreased with increased exposure to the tools, from 35 with the first mathlet to only 13 on the last assignment. Most made comments such as "Generally, the visuals were self explanatory enough to figure out on my own after messing around." In particular, most of those noted that only minor help was needed which usually entailed an interaction with a peer.

Table 2 illustrates the students' responses to the question "How enjoyable was the visual to use?" The participants were asked to give the first five tools a whole number rating on a scale from 1 to 5 , with 1 being "tedious," 3 being "average for an MIT problem set," and 5 being "enjoyable." Again, the scale was revised to a 1 to 7 scale for the next five tools, with 1 being "tedious," 4 being "average for an

Table 1. Responses: What was the Overall Ease of Learning to use the Visual?

\begin{tabular}{|c|c|c|c|c|c|c|c|}
\hline \multirow[t]{2}{*}{ Mathlet } & \multicolumn{7}{|c|}{$\underline{\text { Rating }}$} \\
\hline & 1 & 2 & 3 & 4 & 5 & 6 & 7 \\
\hline Phase lines & 1 & 8 & 13 & 21 & 16 & $\mathrm{n} / \mathrm{a}$ & $\mathrm{n} / \mathrm{a}$ \\
\hline Complex roots & 1 & 10 & 12 & 19 & 13 & $\mathrm{n} / \mathrm{a}$ & $\mathrm{n} / \mathrm{a}$ \\
\hline Amp. \& Phase: 1st order & 2 & 9 & 16 & 17 & 11 & $\mathrm{n} / \mathrm{a}$ & $\mathrm{n} / \mathrm{a}$ \\
\hline Damped vibrations & 1 & 5 & 13 & 22 & 15 & $\mathrm{n} / \mathrm{a}$ & $\mathrm{n} / \mathrm{a}$ \\
\hline Amp. \& Phase: 2nd order & 0 & 4 & 10 & 18 & 24 & $\mathrm{n} / \mathrm{a}$ & $\mathrm{n} / \mathrm{a}$ \\
\hline Beats & 2 & 2 & 6 & 12 & 18 & 9 & 5 \\
\hline Fourier coefficients & 1 & 5 & 6 & 9 & 19 & 10 & 2 \\
\hline Convolution: accumulation & 1 & 2 & 9 & 10 & 14 & 11 & 0 \\
\hline Convolution: flip and drag & 1 & 2 & 8 & 10 & 16 & 10 & 0 \\
\hline Linear phase portraits & 1 & 1 & 4 & 8 & 9 & 13 & 10 \\
\hline
\end{tabular}


Table 2. Responses: How enjoyable was the visual to use?

\begin{tabular}{|c|c|c|c|c|c|c|c|}
\hline \multirow[t]{2}{*}{ Mathlet } & \multicolumn{7}{|c|}{ Rating } \\
\hline & 1 & 2 & 3 & 4 & 5 & 6 & 7 \\
\hline Phase lines & 4 & 7 & 18 & 24 & 6 & $\mathrm{n} / \mathrm{a}$ & $\mathrm{n} / \mathrm{a}$ \\
\hline Complex roots & 6 & 6 & 17 & 22 & 5 & $\mathrm{n} / \mathrm{a}$ & $\mathrm{n} / \mathrm{a}$ \\
\hline Amp. \& Phase: 1st order & 5 & 3 & 15 & 27 & 5 & $\mathrm{n} / \mathrm{a}$ & $\mathrm{n} / \mathrm{a}$ \\
\hline Damped vibrations & 4 & 5 & 16 & 22 & 11 & $\mathrm{n} / \mathrm{a}$ & $\mathrm{n} / \mathrm{a}$ \\
\hline Amp. \& Phase: 2nd order & 2 & 4 & 19 & 21 & 10 & $\mathrm{n} / \mathrm{a}$ & $\mathrm{n} / \mathrm{a}$ \\
\hline Beats & 1 & 3 & 8 & 26 & 9 & 3 & 2 \\
\hline Fourier coefficients & 2 & 2 & 4 & 16 & 10 & 9 & 8 \\
\hline Convolution: accumulation & 4 & 5 & 8 & 20 & 9 & 2 & 1 \\
\hline Convolution: flip and drag & 2 & 2 & 5 & 19 & 15 & 5 & 1 \\
\hline Linear phase portraits & 1 & 2 & 3 & 9 & 13 & 15 & 6 \\
\hline
\end{tabular}

MIT problem set," and 7 being "enjoyable." Fiftynine, 56, 55, 58, 56, 52, 51, 49, 49 and 49 responses were received for the mathlets listed respectively. The table shows these responses in terms of the quantity of each whole number rating received for each tool.

The table shows that most of the mathlets ended up with 50 percent or more of their ratings as "better than an average problem set." Beats and Convolution: Accumulation however did not, with only 27 percent and 24 percent of the ratings as "better than average." Student comments offered some insight into these low ratings:

\footnotetext{
"Beats was a simple visual. It was good, but not as much fun to play with. (Not much happens in this one besides when resonance is reached.)"

"The time spent on Convolution: Accumulation caused it to be somewhat tedious. If I had been able to understand it within a reasonable amount of time I would have found it enjoyable."

"I was very happy to understand what I did, but it took me too long-It would have been just as helpful if there has been some further explanation of the different graphs on the page.'

"Convolution Accumulation was just weird, and not much explanation was presented in lecture to help with it in terms of the topic."
}

The enjoyment ratings for the first five mathlets were further examined by relating them to the respondents' performance on the first semester exam, the results presented in Table 3 . It was decided to only look at the first five mathlets as they provided a consistent rating scale while avoiding the outlier ratings from Beats and Convolution: Ahead. The 60 participants were sorted into three different groups, 16 that scored below 70 on the exam, 21 that scored in the range of 70-85, and 23 that received a grade of 86 or above.

Notably, those in the lowest-scoring exam group gave the highest ratings for each tool. While 63 percent of the ratings in this group were a 4 or 5 for Phase Lines, only 52 percent of the ratings in the middle-scoring group and only 36 percent of the ratings in the highestscoring exam group were a 4 or 5. For Complex Roots, 60 percent of the ratings in the lowest-scoring group were a 4 or 5 while only 45 percent of the ratings in the middle-scoring group and only 43 percent of the ratings in the highest-scoring exam group were a 4 or 5 . Amplitude and Phase: First Order had 73 percent "above average" ratings in the lowest-scoring group, 58 percent in the middle-scoring group and only 48 percent in the highest-scoring exam group. Damped Vibrations had 73 percent "above average" ratings in the lowest-scoring group, 50 percent in the middlescoring group and 52 percent in the highest-scoring exam group. Amplitude and Phase: Second Order had 67 percent "above average" ratings in the lowestscoring group, but only 52 percent in both the middlescoring and highest-scoring exam group.

Table 4 illustrates the student's responses to the question "How much did each visual help you to understand the topic illustrated?" The participants were asked to give the first five tools a whole number rating on a scale from 1 to 5 , with 1 being "a waste of time" and 5 being "really made it clear." Again, the scale was revised to a 1 to 7 scale for the next five tools, with 1 being "a waste of time" and 7 being "really made it clear." Sixty, 56, 55, 58, 57, 51, 50, 48, 48 and 49 responses were received for the mathlets listed respectively. The table shows these responses in terms of the quantity of each whole number rating received for each tool. 
Table 3. Responses: How enjoyable was the visual to use?

\begin{tabular}{|c|c|c|c|c|c|c|}
\hline & \multirow[t]{2}{*}{ Mathlet } & \multicolumn{5}{|c|}{ Rating } \\
\hline & & 1 & 2 & 3 & 4 & 5 \\
\hline \multirow[t]{5}{*}{$0-69$} & Phase lines & 1 & 1 & 4 & 8 & 2 \\
\hline & Complex roots & 1 & 1 & 4 & 8 & 1 \\
\hline & Amp. \& Phase: 1st order & 2 & 1 & 1 & 9 & 2 \\
\hline & Damped vibrations & 1 & 1 & 2 & 6 & 5 \\
\hline & Amp. \& Phase: 2nd order & 1 & 0 & 3 & 4 & 4 \\
\hline \multirow[t]{5}{*}{$70-85$} & Phase lines & 1 & 1 & 8 & 9 & 2 \\
\hline & Complex roots & 3 & 1 & 7 & 7 & 2 \\
\hline & Amp. \& Phase: 1st order & 0 & 1 & 7 & 9 & 2 \\
\hline & Damped vibrations & 0 & 2 & 8 & 7 & 3 \\
\hline & Amp. \& Phase: 2nd order & 0 & 1 & 9 & 7 & 4 \\
\hline \multirow[t]{5}{*}{$86-100$} & Phase lines & 2 & 5 & 7 & 6 & 2 \\
\hline & Complex roots & 2 & 4 & 6 & 7 & 2 \\
\hline & Amp. \& Phase: 1st order & 3 & 1 & 7 & 9 & 1 \\
\hline & Damped vibrations & 3 & 2 & 6 & 9 & 3 \\
\hline & Amp. \& Phase: 2nd order & 1 & 3 & 7 & 10 & 2 \\
\hline
\end{tabular}

Table 4. Responses: How much did each visual help you to understand the topic illustrated?

\begin{tabular}{|c|c|c|c|c|c|c|c|}
\hline \multirow[t]{2}{*}{ Mathlet } & \multicolumn{7}{|c|}{ Rating } \\
\hline & 1 & 2 & 3 & 4 & 5 & 6 & 7 \\
\hline Phase lines & 6 & 9 & 19 & 19 & 7 & $\mathrm{n} / \mathrm{a}$ & $\mathrm{n} / \mathrm{a}$ \\
\hline Complex roots & 9 & 13 & 11 & 13 & 10 & $\mathrm{n} / \mathrm{a}$ & $\mathrm{n} / \mathrm{a}$ \\
\hline Amp. \& Phase: 1st order & 4 & 6 & 19 & 24 & 2 & $\mathrm{n} / \mathrm{a}$ & $\mathrm{n} / \mathrm{a}$ \\
\hline Damped vibrations & 4 & 7 & 16 & 21 & 10 & $\mathrm{n} / \mathrm{a}$ & $\mathrm{n} / \mathrm{a}$ \\
\hline Amp. \& Phase: 2nd order & 4 & 7 & 19 & 23 & 4 & $\mathrm{n} / \mathrm{a}$ & $\mathrm{n} / \mathrm{a}$ \\
\hline Beats & 1 & 2 & 12 & 13 & 14 & 6 & 3 \\
\hline Fourier coefficients & 1 & 5 & 6 & 9 & 19 & 10 & 2 \\
\hline Convolution: accumulation & 3 & 8 & 6 & 12 & 13 & 3 & 1 \\
\hline Convolution: flip and drag & 1 & 1 & 8 & 11 & 14 & 9 & 2 \\
\hline Linear phase portraits & 1 & 0 & 1 & 4 & 7 & 13 & 23 \\
\hline
\end{tabular}

The data shown indicates that for each mathlet, more than 60 percent of the participants gave the first five tools a rating of 3,4 or 5 and the last five tools a rating of 4, 5, 6 or 7 . Thus, more that 60 percent of the participants found the tools "average or better" in aiding in their understanding. Specifically, in the case of the Linear Phase Portraits, only 4 percent of the participants found the mathlet "below average" in helping their understanding.

The understanding ratings for the first five mathlets were also related to the respondents' learning style, the results presented in Table 5. Students who identified themselves with learning style A prefer to see material first presented visually. Students who identified themselves with learning style B prefer to have material first presented computationally. Students who identified themselves with learn- ing style $\mathrm{C}$ prefer to have material first presented theoretically.

Not unexpectedly, the first group reported higher gains in understanding from using the mathlets than did the other two groups. While 72 percent of the ratings in this group were a 4 or 5 for Phase Lines, only 29 percent of the ratings in group B and 33 percent of the ratings in the group $\mathrm{C}$ were a 4 or 5 . For Complex Roots, 62 percent of the ratings in group A were a 4 or 5 while only 30 percent in group $\mathrm{B}$ and 35 percent in group $\mathrm{C}$ were a 4 or 5 . Amplitude and Phase: First Order had 82 percent "above average" ratings in group A, 33 percent in group B and only 29 percent in group C. Damped Vibrations had 72 percent "above average" ratings in group A, 48 percent in group B and 41 percent in group C. Amplitude and Phase: Second Order had 71 percent 
Table 5. Responses: How much did each visual help you to understand the topic illustrated?

\begin{tabular}{|c|c|c|c|c|c|c|}
\hline & \multirow[t]{2}{*}{ Mathlet } & \multicolumn{5}{|c|}{ Rating } \\
\hline & & 1 & 2 & 3 & 4 & 5 \\
\hline \multirow[t]{5}{*}{ Style A } & Phase lines & 0 & 2 & 3 & 9 & 4 \\
\hline & Complex roots & 0 & 5 & 1 & 7 & 3 \\
\hline & Amp. \& Phase: 1st order & 0 & 1 & 2 & 14 & 0 \\
\hline & Damped vibrations & 0 & 2 & 3 & 7 & 6 \\
\hline & Amp. \& Phase: 2 nd order & 0 & 2 & 3 & 11 & 1 \\
\hline \multirow{5}{*}{ Style B } & Phase lines & 3 & 5 & 9 & 6 & 1 \\
\hline & Complex roots & 6 & 4 & 6 & 3 & 4 \\
\hline & Amp. \& Phase: 1st order & 3 & 2 & 9 & 5 & 2 \\
\hline & Damped vibrations & 2 & 3 & 7 & 8 & 3 \\
\hline & Amp. \& Phase: 2nd order & 2 & 5 & 9 & 5 & 3 \\
\hline \multirow[t]{5}{*}{ Style C } & Phase lines & 3 & 2 & 7 & 4 & 2 \\
\hline & Complex roots & 3 & 4 & 4 & 3 & 3 \\
\hline & Amp. \& Phase: 1st order & 1 & 3 & 8 & 5 & 0 \\
\hline & Damped vibrations & 2 & 2 & 6 & 6 & 1 \\
\hline & Amp. \& Phase: 2 nd order & 2 & 0 & 7 & 7 & 0 \\
\hline
\end{tabular}

"above average" ratings in group A, but only 33 percent in group B and 44 percent in group C.

Further general comments on survey results. These artistic special purpose tools were welcomed by students much more readily than Matlab exercises. This was true even when the Matlab exerices were mediated by a special purpose graphical user interface. As one student commented, "Sheer joy compared to 18.02 [Vector Calculus] Matlab assignments."

Students spent between 15 and $30 \mathrm{~min}$ on each mathlet, and they felt that this was reasonable. A student said, "I feel like the time was well-spent since I learned a lot during the time." Many students commented that working on these computer manipulatives was a welcome respite from the standard paper and pencil homework. The instructors thought the applets stimulated students to spend more time on task. Several students remarked, "I genuinely enjoyed using these visuals [as the mathlets were being called at the time] and had no problem spending a lot of time on them." and "I spent more time on it than what was just required to complete the assignment because I found it interesting."

There is considerable discussion in the literature about how simulations should be timed relative to other treatments of the same material: see Lipson (2006), for example. Homework requiring the use of one of the mathlets fell due before it was lectured on in class: the Linear Phase Portrait mathlet. Several students made comments supporting the following one: "These were great visuals, they were instrumental in helping me begin to understand how phase portraits look and it helped me to understand the following lectures."

The first implementations were presented as platform specific executables, and students had to go to an Athena cluster to use them. This was deeply unpopular among the students, and we worked to port the mathlets to Java so students could work on them anywhere, but we feel that there was a real advantage to bringing students to a common location to work on these projects. It encouraged group work and peer discussion. This became apparent from the responses to questions about how the students got help when they needed it: "Someone else in the Athena cluster working on the same thing," and "Friends working on the pset [problem set] in the same cluster."

It is also interesting to note that various student comments point towards the tools being successful in dealing with the three teaching dilemmas presented earlier. Here is a sample of these comments.

\section{Facilitation of transfer}

"I found my biggest problem was that I didn't really understand what the graphs meant from class/reading. Thus, playing with the graph and having to use them really forced me to understand them, and I would DEFINITELY recommend continuing to use them. It's funny that I didn't even realize how much they help until you are trying to think about it later and you remember the graph."

\section{Example versus Theory}

"I have always thought that the visuals where the most fun part of the problem set. It is always satis- 
fying to see that the experimental value is not too far off from the theoretical value"

"The concept of nth roots was abstract before the visual, but was clear afterwards."

"The best value I've gotten out of any of them was the PhLines with its two graphs of the response and $\mathrm{f}(\mathrm{y})$ in $\mathrm{dy} / \mathrm{dx}=\mathrm{f}(\mathrm{y})$. That solidified my intuition of what made a stable point and how adding or subtracting constants could be interpreted."

"Helpful to see how constants affect graph, also helpful to see how function behaves around the facts that you're trying to find - w/out visuals, would only see the one pt calculated; w/visuals, see entire curve, get used to their behavior."

\section{Algorithm versus Concept}

"Sometimes just calculating with something is not very effective in helping to understand it. I think the visuals were very good, in general, in helping me understand concepts and think about concepts rather than just follow a standard method of solving a problem."

\begin{abstract}
"The damped vibes visual really helped my understanding of the concept of under damping and over damping. The professor gave a great explanation in lecture, but I was not really sure how the values for different variables would make a difference. The ability to manipulate these variables helped me to see what damping relates to."

"I had no idea what damping was. Playing around with the damping visual I saw that without damping, the function just oscillated with the same amplitude. If someone had told me this, it wouldn't have meant anything to me. I had to see it."
\end{abstract}

\section{CONCLUSION}

Summary of Results. We have employed an integrated approach, involving educational assessment from the outset, to the development of a suite of iterative simulations supported by guided instructional support for use in a university level ordinary differential equations course oriented towards engineering majors.

The results of the assessment appear to show that on the whole, students found the mathlets easy to use. The students generally enjoyed using the tools, especially the lower-performing students, so it seems possible that they served as a motivational tool for the struggling student. Not unexpectedly, visual learners found the tools most useful in aiding their understanding. The other learning types, however, still found the mathlets to be more helpful than not in assisting in their understanding of the relevant topics. Various student comments support the notion that the tools are useful in facilitating transfer as well as dealing with the teaching dilemmas of example versus theory and algorithm versus concept discussed earlier.

It is important to note that the mathlets are not intended to replace textbooks or teachers. And of course, they were not viewed as beneficial by everyone as there were students that gave low ratings to the tools and offered less than positive comments. Taken as a whole however, the mathlets should be seen as a valuable resource to be used alongside textbooks and lectures in promoting retention and understanding in the learning of differential equations.

The Promise of Technology. We will end with some general observations about the use of computers in teaching. These principles combine design principles we had in mind at the outset with things we learned in the course of this work. The earlier sections have effectively laid out our response to these principles.

The promise of computers as an educational tool has been trumpeted ever since the earliest days of the computer revolution. The following is a recent example (Bransford et al. (2000), p. 215):

\begin{abstract}
Some scholars assert that simulations and computerbased models are the most powerful resources for the advancement and application of mathematics and science since the origins of mathematical modeling during the Renaissance. The move from a static model in an inert medium, like a drawing, to dynamic models in interactive media that provide visualization and analytic tools is profoundly changing the nature of inquiry in mathematics and science.
\end{abstract}

The rapid increase in power, flexibility, and ubiquity of computers has increased this potential. Just as important, however, is the insight we have gained into the limitations and optimal use of technology. We list some observations gleaned from the work outlined in this paper and many sources-for example, Breslow (2006) - and indicate how they have influenced the design and use of the dAIMP Mathlets. ${ }^{3}$

(1) Technology is most effective when it meets a need and fits naturally into the overall educational context. Absent these conditions it can be a distraction.

(2) Use of technology involves a change in what is taught, not just in how it is taught. For exam-

\footnotetext{
${ }^{3}$ This list is a modification of one developed by the first author in a white paper presented to the MIT Task Force on the Undergraduate Commons in December, 2005.
} 
ple, it is often possible to stress conceptual points more if direct graphical representations are easily available. Computational power brings a range of otherwise inaccessible examples into play.

(3) Student use of technology needs to be highly structured. Free discovery can work on a simulation substrate but tends to be ineffective unless it is done in a group setting.

(4) There is a high startup cost. Programming is expensive. As a result, the cost of altering functioning technological components can exert a conservative force on curricular development.

(5) There is a price paid by both student and teacher in learning to use the specific conventions attached to the technology. This has been a problem in integrating computational languages such as Matlab into basic courses, and it can discourage faculty from adopting even highly focused teaching objects.

(6) Aesthetics matter. Students use a device more willingly and profitably if it is attractive, simple, intuitive, and convenient.

(7) Information technology offers new avenues for enhancing communication. Email and websites have become ubiquitous. Online tutors are increasingly sophisticated at providing instantaneous feedback to students. Much can be learned about student understanding from logging various responses (via PRS to concept quizzes, for example).

\section{ACKNOWLEDGEMENTS}

The design and construction of this courseware was made possible by a generous grant from the Alex and Britt d'Arbeloff Fund for Excellence. Hu Hohn, Director of the Computer Arts Center at Massachusetts College of Art, collaborated with the first author in the design of these programs, and did the initial coding of them. The translation to Java was begun by Ashot Heyrapetyan and completed by Jean-Michel Claus. The second author was supported by the MIT Teaching and Learning Laboratory. This paper is a revised and expanded version of a paper presented at the annual meeting of the
American Educational Research Association held in San Francisco, California, from April 7-11, 2006.

\section{REFERENCES}

Adamczyk, B., Reffeor, W., and Jack, H. (2002). Math literacy and proficiency in Engineering students. Proceedings of the 2002 American Society for Engineering Education Annual Conference and Exposition.

Alessi Trollip, S. M. S. R. (2001). Multimedia for learning: Methods and development, Allyn and Bacon, Boston.

Bransford, J. D., Brown, A. L., and Cocking, R. R. (2000). How People Learn: Brain, Mind, Experience, and School, Expanded Edition, National Academy Press, Washington, DC.

Bransford, J. D., and Schwartz, D. L. (1999). Rethinking transfer: A simple proposal with multiple implications. In A. P. D. IranNejad Pearson (Ed.Review of research in education (pp. 61100).24 Washington, DC: American Educational Research Association.

Breslow, L. (2006). Lessons Learned: Findings from an MIT initiative in educational technology $(2000-2005)$. Journal of Science Education and Technology 16, in press.

Edwards, P., and Edwards, P. M. (2003). Tackling the mathematics problem with MathinSite. Global Journal of Engineering Education 7(1): 95-102.

Killian, J. R. (1949). The Inaugural Address. Retrieved on July 23, 2006 from http://libraries.mit.edu/archives/exhibits/inaugurations/killian.html.

Laurillard, D. (2002). Rethinking University Education: A conversational framework for the effective use of learning technologies. 2nd edn., RoutledgeFalmer.

Lipson, A. (2006). The impact of computer simulations on student learning in science: a view from the literature. Teaching and Learning Laboratory, MIT.

Mayer, R. E. (2004). Should there be a three-strikes rule against pure discovery learning: the case for guided methods of instruction. American Psychologist 59(1): 14-19.

McDill, J., Rash, A., and West, B. (1997). Interactive computer illustrations and multimedia: new enhancements for calculus and differential equations courses. Paper presented at the Third International Conference on Technology in Mathematics Teaching. Koblenz, Germany.

Miller, H., and Upton, D. (2002). Personal interviews with M.I.T. faculty.

Nasr, R., Hall, S., and Garik, P. (2003). Student misconceptions in signals and systems and their origins. Paper presented at the $33 \mathrm{rd}$ ASEE/IEEE Frontiers in Education Conference. Boulder, CO.

Schwartz, D. L., Bransford, J., and Sears, D. (2005). In J. Mestre (Ed.Transfer of Learning from a Modern Multidisciplinary Perspective (pp. 1-51). Greenwich, CT: Information Age Publishing.

Upton, D. (2001). M.I.T. student focus group interview.

West, B., Strogatz, S., McDill, J. M., Cantwell, J., and Hohn, H. (1997). Interactive Differential Equations. Addison Wesley Longman.

Willcox, K., and Bounova, G. (2004). Mathematics in engineering: Identifying, Enhancing and linking the implicit mathematics curriculum. Proceedings of the 2004 American Society for Engineering Education Annual Conference \& Exposition. Available at http://me.nmsu.edu/ aseemath/papers.html in Session 2465 Curriculums in Transition. 